\title{
Mass producing an efficient NIR spectrograph
}

John C. Wilson, Charles P. Henderson, Terry L. Herter, Keith Matthews, Michael F. Skrutskie, et al.

John C. Wilson, Charles P. Henderson, Terry L. Herter, Keith Matthews, Michael F. Skrutskie, Joseph D. Adams, Dae-Sik Moon, Roger Smith, Nick Gautier, Michael Ressler, B. T. Soifer, Sean Lin, James Howard, John LaMarr, Todd M. Stolberg, Jeff Zink, "Mass producing an efficient NIR spectrograph," Proc. SPIE 5492, Ground-based Instrumentation for Astronomy, (30 September 2004); doi: 10.1117/12.550925

Event: SPIE Astronomical Telescopes + Instrumentation, 2004, Glasgow, United Kingdom 


\title{
'Mass Producing' an efficient NIR spectrograph
}

\author{
J. C. Wilson ${ }^{a}$, Charles P. Henderson ${ }^{b}$, Terry L. Herter ${ }^{b}$, K. Matthews $^{c}$, M. F. Skrutskie ${ }^{a}$, \\ Joseph D. Adams ${ }^{b}$, Dae-Sik Moon ${ }^{c}$, Roger Smith ${ }^{c}$, Nick Gautier ${ }^{d}$, Mike Ressler ${ }^{d}$, B. T. Soifer ${ }^{c}$, \\ Sean $\operatorname{Lin}^{c}$, J. Howard ${ }^{e}$, J. LaMarr ${ }^{e}$, T. M. Stolberg ${ }^{e}$, and J. Zink ${ }^{e}$ \\ ${ }^{a}$ University of Virginia, 530 McCormick Rd, Charlottesville, VA, 22903, USA; \\ ${ }^{b}$ Cornell University, Space Sciences Bldg, Ithaca, NY, 14853, USA; \\ ${ }^{c}$ Caltech, Div of Physics, Mathematics, \& Astronomy, MS 320-47, Pasadena, CA, 91125, USA; \\ ${ }^{d}$ Jet Propulsion Laboratory, MS 169-506, 4800 Oak Grove Dr, Pasadena, CA, 91109, USA; \\ ${ }^{e}$ Axsys IR Systems, 152 Rangeway Road, North Billerica, MA, 01862, USA
}

\begin{abstract}
Four institutions are collaborating to design and build three near identical $\lambda / \Delta \lambda \sim 2700$ cross-dispersed nearinfrared spectrographs for use on various 5-10 meter telescopes. The instrument design addresses the common observatory need for efficient, reliable near-infrared spectrographs through such features as broad wavelength coverage across 6 simultaneous orders $(0.8-2.4 \mu \mathrm{m})$ in echelle format, real-time slit viewing through separate optics and detector, and minimal moving parts. Lastly, the collaborators are saving money and increasing the likelihood of success through economies of scale and sharing intellectual capital.
\end{abstract}

Keywords: Near Infrared, Spectroscopy, Cross-Dispersed

\section{INTRODUCTION}

The availability of efficient moderate resolution near-infrared (NIR) spectrographs with wide wavelength coverage at modern telescopes is increasingly important for such work as investigating targets identified in large-scale imaging surveys including the Two Micron All-Sky Survey ${ }^{1}$ (2MASS) and Sloan Digital Sky Survey ${ }^{2}$ (SDSS). These targets can be faint in the visible but bright in the NIR, such as brown dwarfs and dust obscured galaxies. These spectrographs are also useful for the spectral follow-up of objects identified in the mid-infrared by e.g. Spitzer Space Telescope ${ }^{3}$ and soon the Stratospheric Observatory for Infrared Astronomy (SOFIA). They are also important for studying high redshift objects with visible rest frame diagnostic lines that have been red-shifted into the near-infrared.

Cornell and JPL sought to design a cost-effective seeing-limited spectrograph that would excel in this spectral follow-up or 'triage' role for the Palomar Observatory 200-inch telescope, for which there is no existing facility near-infrared spectrograph. We use the term 'triage' because we envision astronomers using the spectrograph to rapidly and efficiently collect spectra of a list of hitherto unknown targets culled from e.g. 2MASS or Spitzer Space Telescope databases. Because telescope time, especially for large apertures, is a scarce resource, this 'triage' capability is maximized by providing as wide a simultaneous wavelength coverage as possible. Observing efficiency is increased compared to spectrographs that require multiple instrument settings, e.g. grating rotations or grism changes to achieve the same coverage. In addition, the stitching of adjacent spectral regions that overlap is also improved when the various spectral regions are imaged simultaneously on the same detector. Lastly, a separate NIR slit-viewing channel with wide field of view (FOV) is crucial for field identification and keeping an object in the slit in real-time.

Paradoxically, there is a relative lack of instruments of this kind at modern telescopes. This led Caltech and the University of Virginia to join Cornell and JPL in the design and fabrication of three near-identical efficient, cost effective moderate resolution seeing limited near-infrared spectrographs. Cornell and JPL are building a copy to be a facility instrument for the Palomar 200-inch telescope. Caltech is building a copy to be a facility instrument for the Keck 10-meter telescopes. And the University of Virginia is building a copy to be a visiting instrument at 5-10 meter telescopes.

Correspondence: E-mail: jcw6z@virginia.edu, Telephone: 1 (434) 924-4907 


\section{MULTI-INSTITUTIONAL TEAMING}

The benefits of multi-institutional teaming to build near-identical copies of an instrument are real and both tangible and intangible. Financially the partners save money through 'volume' purchases of some of the instrument components. For example, unit costs for the spectrograph camera opto-mechanical assembly was discounted $30 \%$ when three units were purchased instead of one. Similar savings are expected for other portions of the instrument, including the Dewar fabrication. The partners also save money through sharing one-time design cost charges, such as design studies for stray light analysis and test AR coating runs.

On the intangible side, the sharing of intellectual capital and institutional experience through previous instrument work is extremely beneficial. We also expect to improve instrument performance through the sharing of lessons learned as the various copies are commissioned.

\section{DESIGN OVERVIEW}

\subsection{Desired Core Capabilities}

- Wide simultaneous wavelength coverage $(0.8-2.4 \mu \mathrm{m})$

- Sufficient slit length for effective sky subtraction through nodding

- Sufficient spectral resolution to allow a significant fraction of pixels along the spectral orders to be free of airglow contamination ${ }^{4}$

- Throughput maximized for K-band, but wavelength coverage to $0.8 \mu \mathrm{m}$ to allow overlap with spectra from red-optical CCD based instruments

- Separate slit viewing channel to enable real-time slit viewing, source identification, and near-infrared guiding

- Accommodate various incoming telescope f/\#'s with customizable reflective re-imaging fore-optics

\subsection{Imposed Constraints}

- Ensure high reliability through minimal use of moving parts

- Keep costs on the order of $\$ 1$ million US

- Develop a compact Dewar design with minimal flexure that can be used at cassegrain, bent-cassegrain and nasmyth ports with little modification

\subsection{Design Trades}

There is obvious tension between the first three core capabilities given a finite availability of pixels, especially since detectors are typically the most expensive material costs in infrared instruments. We balanced our desired capabilities and constraints by concentrating on a design that utilized two adjacent quadrants of a Rockwell

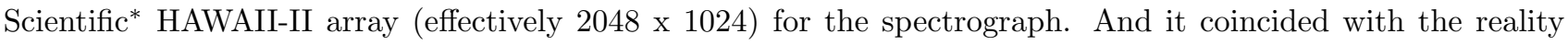
that an excessive number of prisms in series would be required to provide enough cross dispersion to fill 2048 pixels in the slit length direction. We are using three prisms in series to cross disperse 6 orders across 1024 pixels. Prisms provide cross-dispersion instead of a first order grating because our wavelength coverage exceeds a factor of two. We chose to use a grating with first order at $\sim 6.6 \mu \mathrm{m}$ so the third order peak efficiency was well centered on $\mathrm{K}$ band. The blue end of the spectral coverage is driven by the rapidly dropping QE with wavelength at $0.8 \mu \mathrm{m}$ in $\mathrm{HgCdTe}$ detectors. The red end is driven by the edge of the $\mathrm{K}$ atmospheric window. The final design is summarized in Table 1.

\footnotetext{
${ }^{*}$ Rockwell Scientific Company, LLC, 5212 Verdugo Way, Camarillo, CA, USA
} 
Table 1. Instrument Design Summary

\begin{tabular}{|c|c|}
\hline Resolution & 2700 \\
\hline Simultaneous $\lambda$ Coverage & $0.8-2.4 \mu \mathrm{m}$ (across 6 orders) \\
\hline Nominal Slit Size on sky (Palomar 200-inch) & $1.0 \times 30$ arcsec \\
\hline Sampling & 2.7 pix per resolution element (slit width) \\
\hline Spectrograph Detector & 2 quadrants of Hawaii-II (effectively 2048 x 1024) \\
\hline Primary Disperser & Reflection Grating \\
\hline Cross Disperser & ZnSe and 1 Infrasil prism in series \\
\hline Slit Viewer Band & $\mathrm{K}_{\mathrm{s}}$ \\
\hline Slit Viewer Detector & $4 \times 4$ arcmin \\
\hline Slit Viewer FOV (Palomar 200-inch) & \\
\hline
\end{tabular}

\subsection{Same design for different telescopes?}

Various optical and mechanical features enable nearly identical instruments to be used on different telescopes. First, a reflective reimager based upon mis-matched off-axis paraboloids is used to convert a telescope's incoming f/\# to a common f/10.7 at the slit (Section 4.1). Past the telescope focus, the first off-axis paraboloid collimates the beam. After passing through a Lyot stop, another off-axis paraboloid focuses the light upon the slit. An instrument to be used on a telescope with an $\mathrm{f} / 15$ beam would have a different first off-axis paraboloid installed compared to an instrument to be used on a telescope with an $\mathrm{f} / 11$ beam.

Secondly, given a common $\mathrm{f} / 10.7$ beam falling on the slit, the plate scale change with aperture fortuitously matches the improvement in seeing between the Palomar 200-inch (5-meter) telescope and the Keck 10-meter. This allows the same slit and spectrograph camera to serve both telescopes. Specifically, an instrument with a 1 arcsec slit optimized for the Palomar 200-inch (5-meter) telescope, with $\sim 1$ arcsec seeing, scales correctly for use on the Keck 10-meter telescope with $\sim 0.5$ arcsec seeing.

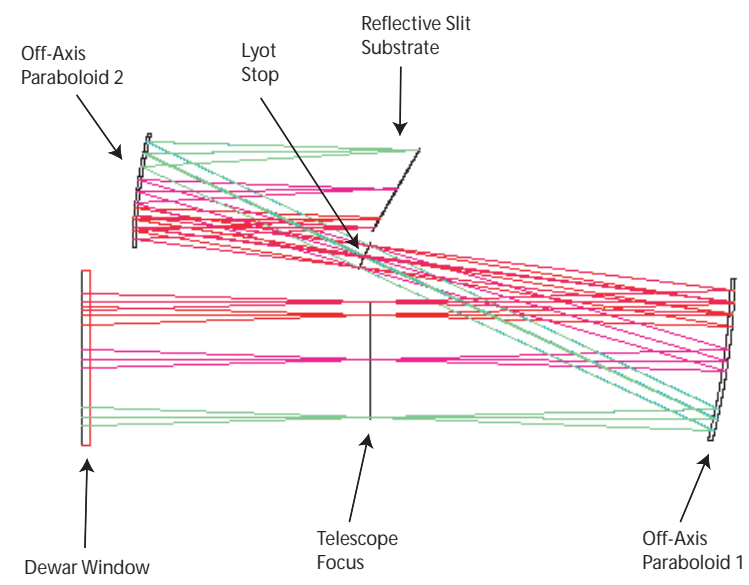

Figure 1. Schematic of the instrument re-imaging optics. f/16 light from the telescope enters through the sapphire Dewar window at the left and immediately comes to a focus. Past the focus the light is reflected and collimated by an off-axis paraboloid. After passing through the Lyot stop, the light is reflected again and focused at $\mathrm{f} / 10.7$ onto the reflective slit. Telescopes of different $\mathrm{f} /$ \# formats can be accommodated by changing the first off-axis paraboloid. 
Lastly, the Dewar cryogen tank and fill tube end point within the tank are designed to allow use of the instrument at cassegrain, bent-cassegrain and nasmyth foci (Section 5.1).

\section{OPTICS}

\subsection{Fore-optics}

The instrument uses a traditional re-imager to produce a cold pupil stop and accomplish f/\# conversion (Figure 1). Light from the telescope enters the Dewar through a square sapphire window and comes to a focus inside the cold volume. A square field stop that defines the slit viewer field is placed at this focus. Past the telescope focus the beam diverges and is collimated by an off-axis paraboloid and folded back towards the front of the Dewar. This off-axis paraboloid is matched to the incoming telescope f/\#. (As discussed in Section 3.4, by simply changing this paraboloid and adjusting the position of the Lyot stop, telescopes with differing f/\#'s can be accommodated.) The collimated beam then passes through a cold Lyot stop placed at the image of the telescope pupil. Lastly the beam is folded back and re-focused at $\mathrm{f} / 10.7$ onto the slit by a second off-axis paraboloid. The slit substrate has not been designed yet but functionally it will be both reflective across the $\sim 4 \times 4$ arcmin slit viewer FOV at the Palomar 200-inch $(\sim 2 \times 2$ arcmin at the Keck 10-meter $)$ and provide for the choice of two or three different slits with varying widths based on user's science desires and seeing. The slit will be tipped $30^{\circ}$ along an axis parallel to the slit length to steer the reflected beam into a separate slit viewing channel.

\subsection{Spectrograph Channel}

The light that is transmitted through the slit diverges at $\mathrm{f} / 10.7$ towards the back of the Dewar and is collimated by another off-axis paraboloid (Figure 2). The resulting collimated beam of $\sim 73 \mathrm{~mm}$ diameter is folded by two

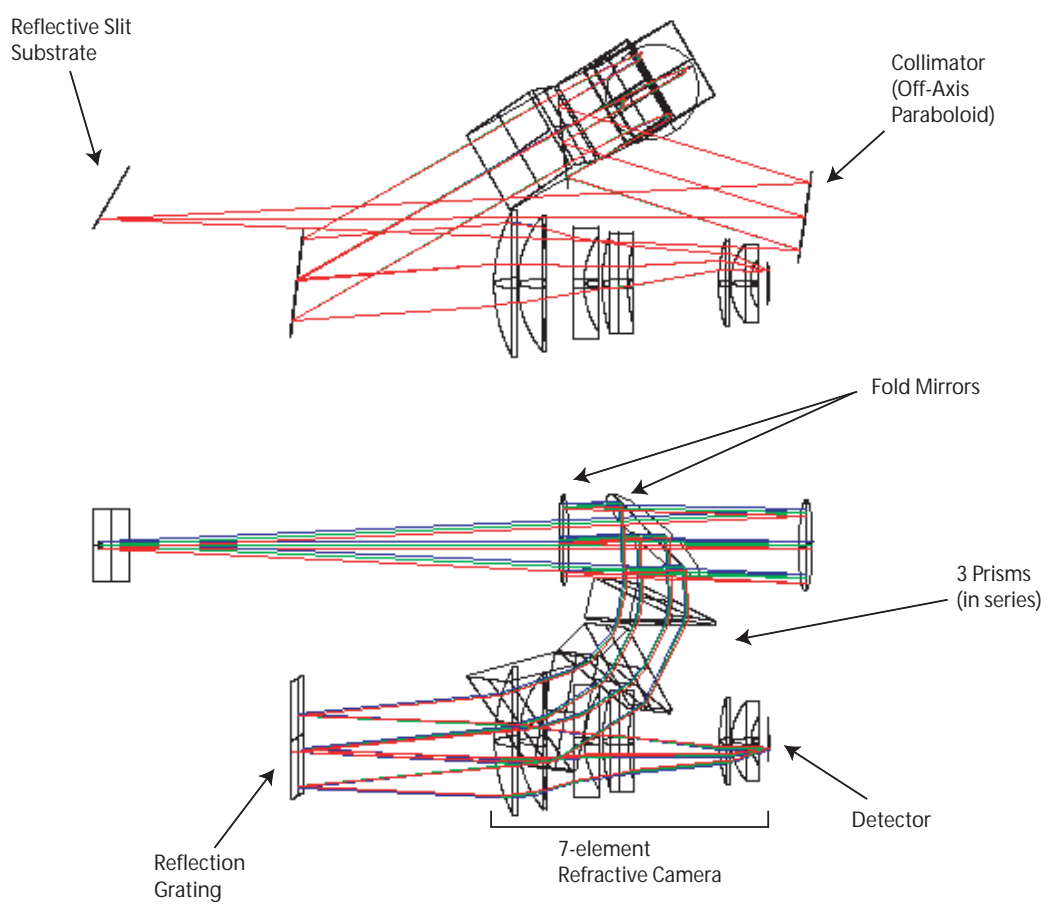

Figure 2. Schematic of the spectrograph portion of the instrument. The schematic is rotated by 90 degrees in the bottom view. Light that passes through the slit is collimated by an off-axis paraboloid and then folded by two flats for packaging purposes. The beam is then pre-dispersed by three prisms in series before reflecting off the grating. Lastly, the dispersed light in re-focused onto two quadrants of a HAWAII-II HgCdTe detector by a 7-element refractive camera. 
flat mirrors towards the pre-dispersing section of the spectrograph. The beam now progresses out of the plane of the fore-optics and spectrograph collimator. Pre-dispersion is accomplished by three prisms in series, two ZnSe prisms with $22^{\circ}$ apex each and one Infrasil prism with $50^{\circ}$ apex. ZnSe and Infrasil have peak partial dispersions near 1 and $2 \mu \mathrm{m}$, respectively. Used in series these materials produce fairly constant separation between the 6 orders covering $0.8-2.4 \mu \mathrm{m}$.

After the prisms a plane reflection grating will disperse the light in the primary dispersion direction. A 110.5 $l / \mathrm{mm}$ grating replica blazed for $6.79 \mu \mathrm{m}\left(22^{\circ}\right.$ blaze) in first order (Littrow configuration) will be purchased from Spectra-Physics ${ }^{\dagger}$ (formerly Richardson Grating Lab). This grating will come from the same master that produced a replica for GNIRS. ${ }^{5}$ The grating will be used with an angular deviation of $30^{\circ}$ (angle between incoming and outgoing beams).

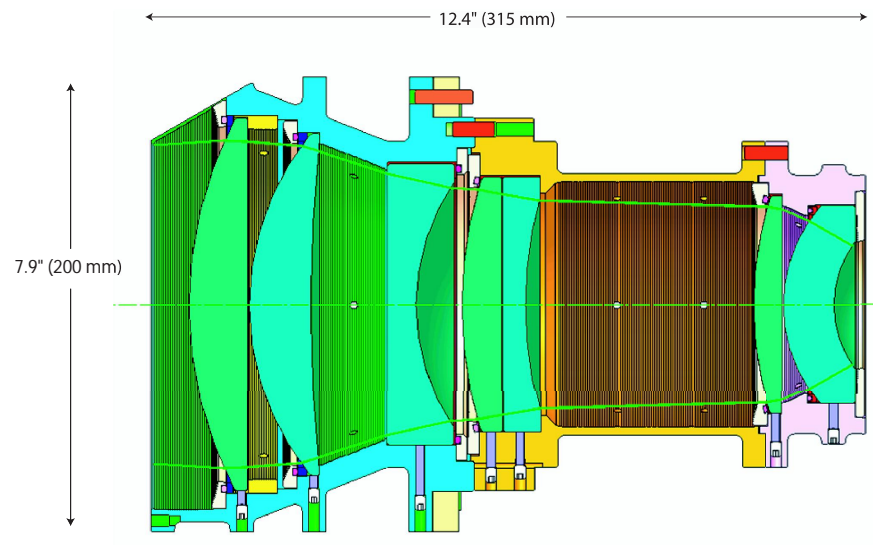

Figure 3. The 7-element f/1.6 spectrograph camera designed and fabricated by Axsys IR Systems. Four materials are used for the elements: ZnSe, Cleartran $(\mathrm{ZnS}), \mathrm{CaF}_{2}$ and Infrasil. The diameter of the first element $\left(\mathrm{CaF}_{2}\right)$ is $164 \mathrm{~mm}$. Two of the element surfaces in the camera are aspheres and one of those is a conic; the remaining surfaces are spherical. A notch is cut from the entrance of the camera mechanical assembly to prevent vignetting of the beam between the prism and grating.

After reflection from the grating, the collimated light is focused onto the detector by a 7-element refractive f/1.6 camera designed by Axsys IR Systems ${ }^{\ddagger}$ (formerly Telic Optics, Inc.). In monochromatic light the camera is $\sim \mathrm{f} / 2.25$ to produce the necessary plate scale of $0.33 \mathrm{arcsec} / \mathrm{pixel}$ at the Palomar 200 -inch $(0.17 \mathrm{arcsec} / \mathrm{pixel}$ at the Keck 10-meter). But the dispersion of the gratings requires a large entrance pupil and results in an f/1.6 system in polychromatic light. The camera utilizes four commonly used NIR materials (ZnSe, Cleartran ${ }^{\S}$ $(\mathrm{ZnS})), \mathrm{CaF}_{2}$ and Infrasil) in a fast 'apochromatic' design to partially correct primary chromatic aberration and minimize secondary spectrum (Figure 3 ). The remaining primary chromatic aberration is taken up by a detector tilt of $\sim 0.9^{\circ}$ along an axis parallel to the spectral orders. Two of the element surfaces in the camera are aspheres and one of those is a conic; the remaining surfaces are spherical.

Six spectral orders are imaged onto two adjacent quadrants of the HAWAII-II detector (Figure 4) to give nearly simultaneous wavelength coverage between $0.8-2.4 \mu \mathrm{m}$ (The only gap in coverage occurs between 1.85 $1.88 \mu \mathrm{m}$, an area of poor atmospheric transmission).

\footnotetext{
${ }^{\dagger}$ Spectra-Physics, 705 St. Paul Street, Rochester, NY 14605 USA Tel: 1 (585) 262-1331.

${ }^{\ddagger}$ Axsys IR Systems, 152 Rangeway Road, North Billerica, MA 01862 USA Tel: 1 (978) 667-4350.

${ }^{\S}$ Cleartran is a water-free form of $\mathrm{ZnS}$ manufactured by Morton International.
} 


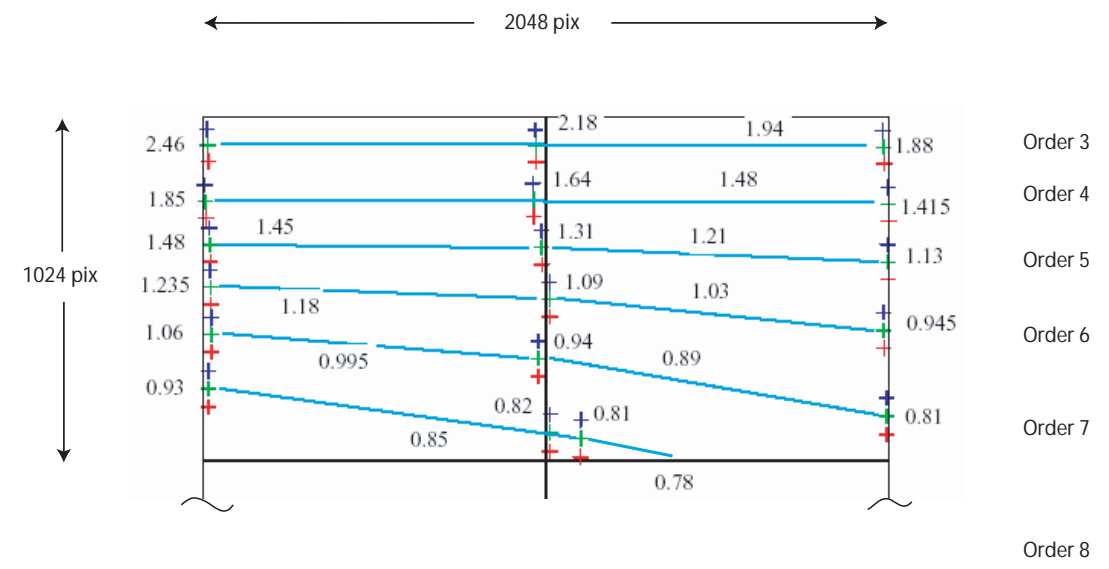

Figure 4. Orders 3 - 8 are imaged onto two adjacent quadrants of a HAWAII-II HgCdTe detector. Slit length is 30 arcsec on the Palomar 200-inch (15 arcsec on the Keck-10m). The sets of three crosses shown at various wavelengths symbolize the top, middle and bottom of the slit.

\subsection{Slit Viewer Channel}

Light that is not transmitted through the slit is reflected by the slit substrate into a separate slit viewing channel (Figure 5). After a fold mirror necessary for packaging, the beam is collimated and re-imaged by a 2-element refractive camera. Both elements are made of Cleartran $(\mathrm{ZnS})$ because the camera is only used in $\mathrm{K}_{\mathrm{S}}$ band $(2.0$ - $2.3 \mu \mathrm{m})$. The first slit viewing lens collimates the beam. A Lyot Stop and $\mathrm{K}_{\mathrm{S}}$ filter are placed at the second image of the telescope pupil, and finally another Cleartran lens re-focuses the light onto a HAWAII-1 HgCdTe detector. Both elements have one surface that is aspheric and one that is flat.

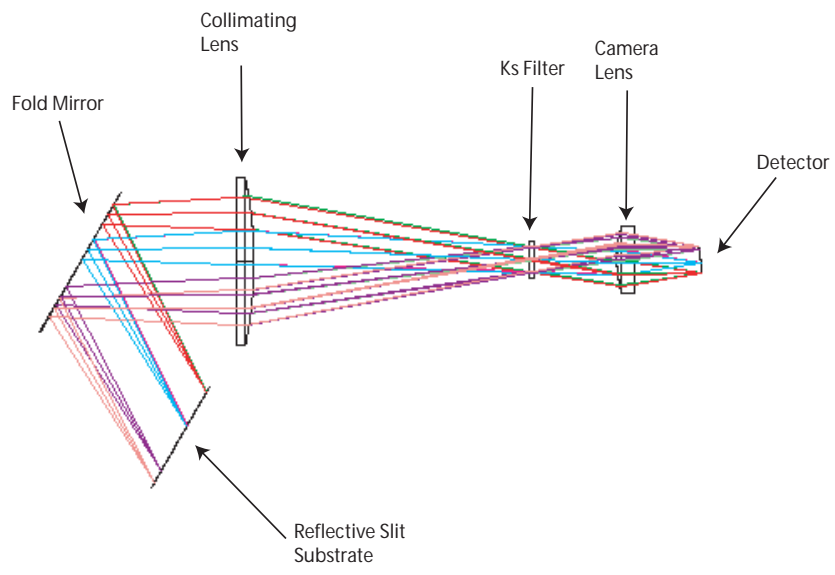

Figure 5. Schematic of the instrument slit viewer optics. Light that is not transmitted through the slit is reflected into a separate slit viewing channel by the slit substrate. After a fold mirror, the beam is collimated through a Cleartran lens, passes through a $\mathrm{K}_{\mathrm{S}}$ filter, and is re-imaged by another Cleartran lens onto a HAWAII-1 HgCdTe detector.

The slit viewing field is used for three purposes: real-time placement of the science object in the slit, field identification, and near-infrared guiding when desired. The image plate scale is $\sim 0.24$ arcsec/pixel at the Palomar 200-inch ( 0.12 arcsec/pixel at the Keck 10-meter) and the FOV is 4 x 4 arcmin at the Palomar 
Table 2. Expected minimum number of suitable guide stars and galaxies in a slit viewer (4 arcmin FOV) pointing with the instrument at the Palomar 200-inch based on 2MASS object counts near the galactic pole.

\begin{tabular}{|c|c|c|c|}
\hline Integration Time $(\mathbf{s e c})$ & $\mathrm{K}_{\mathrm{S}}$ mag $(\mathrm{S} / \mathrm{N}=5)$ & $\mathrm{N}_{\text {Galaxies }}(\mathbf{m i n})$ & $\mathrm{N}_{\text {Stars }}(\mathbf{m i n})$ \\
\hline 1 & 17.2 & 8 & 3 \\
\hline 10 & 18.4 & 42 & 3 \\
\hline 30 & 19.0 & 76 & 3 \\
\hline
\end{tabular}

200-inch (2 x 2 arcmin at the Keck 10-meter). This large FOV was chosen based on 2MASS object counts near the galactic pole to maximize the potential for locating suitable infrared bright guide stars anywhere on the sky (Table 2). The slit is offset from the center of the FOV by 90 arcsec at the Palomar 200-inch (45 arcsec at the Keck 10-meter) (i.e. it is $\sim 30$ arcsec at the Palomar 200-inch ( 15 arcsec at the Keck 10-meter) from the edge of the slit viewer field). Were a suitable guide star not found in the initial slit viewing field, the instrument could be rotated with respect to the telescope. A full rotation would sweep an 8 arcmin field at the Palomar 200-inch (4 arcmin at the Keck 10-meter).

\section{MECHANICS}

\subsection{Cryogenic Dewar}

The optics and detectors will be contained within an $\mathrm{LN}_{2}$ cooled cylindrical Dewar (Figure 6). The cryogen is held in the 'crescent' section of the cylinder. Bulkheads spaced along the length of the cylinder oppose the

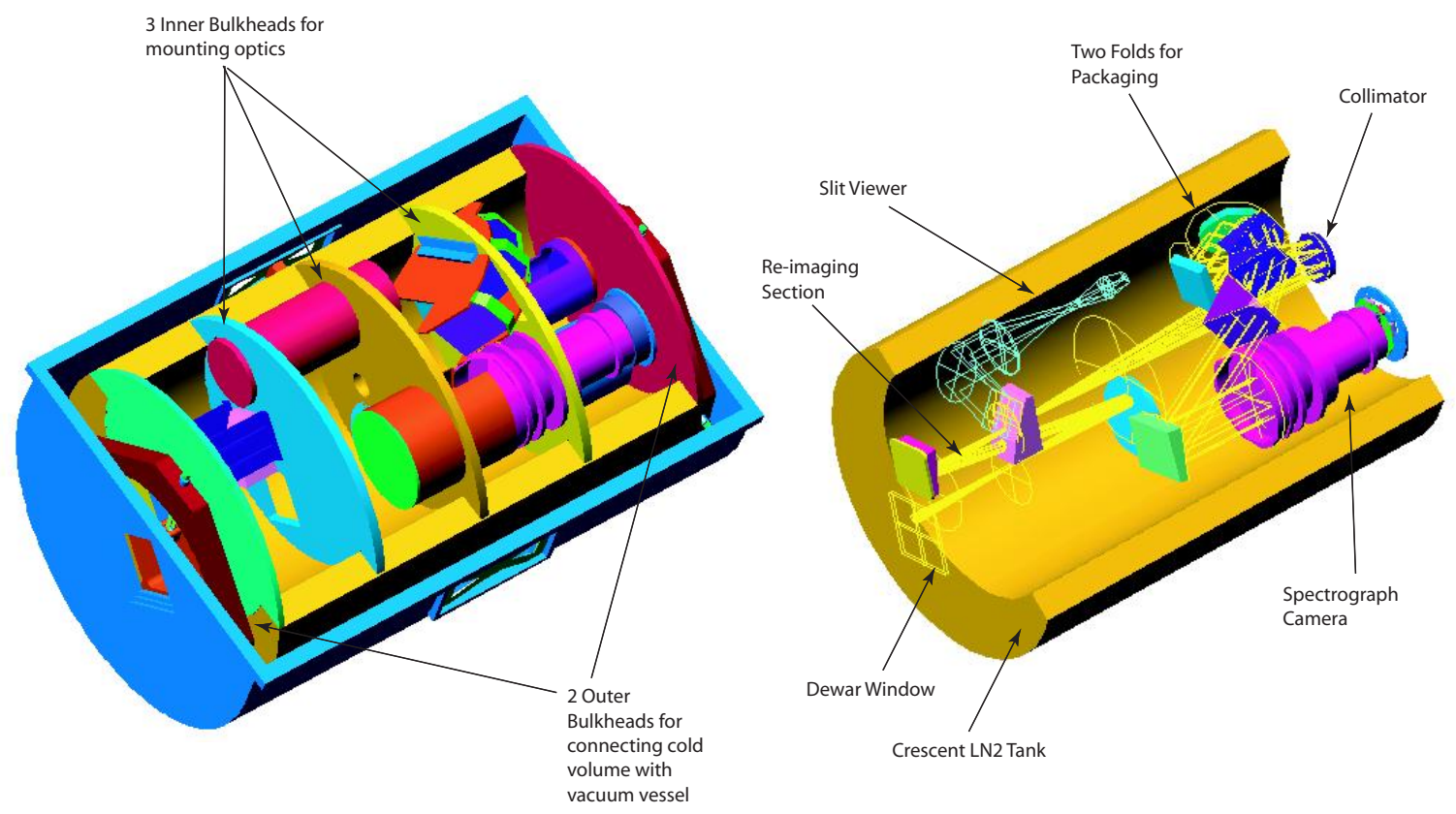

Figure 6. Schematic of the Dewar and opto-mechanical assembly. The cryogen is held in the 'crescent' section of the cylinder. Bulkheads oppose the tendency of the crescent to open when under vacuum and provide a stable framework for mounting optics. The three inner bulkheads supports all of the optics components and the two outer bulkheads provide cold volume mounting points for connection to the vacuum vessel. 


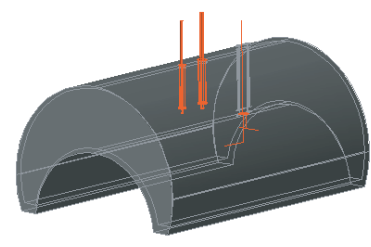

Crescent Shaped $\mathrm{LN}_{2}$ Tank

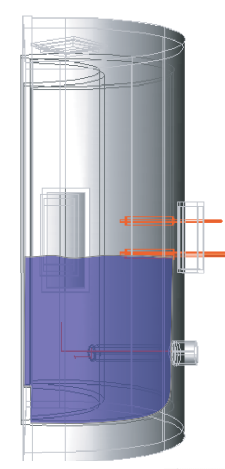

Cassegrain Mounting

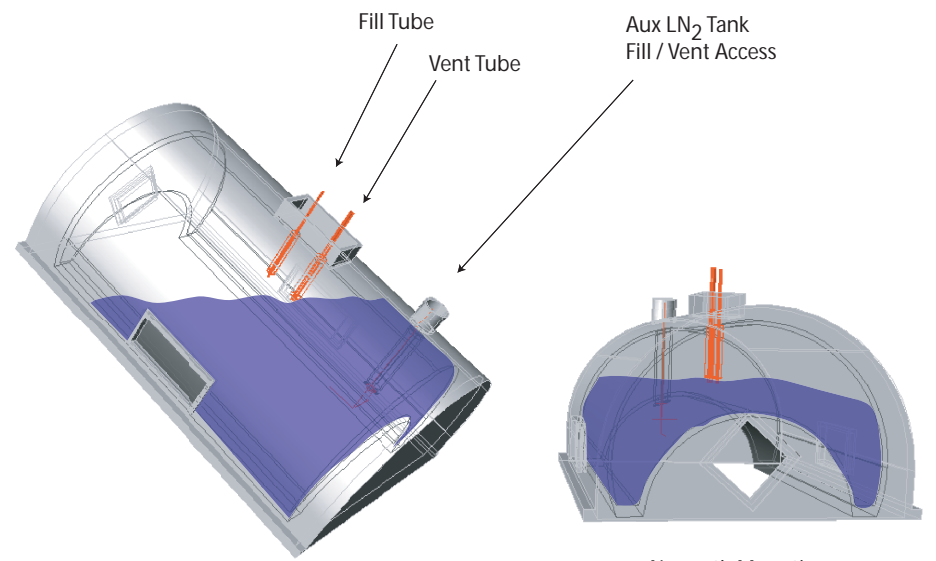

Arbritray

Cassegrain / Bent Cassegrain

Pointing

Figure 7. Schematic of the crescent shaped $\mathrm{LN}_{2}$ tank. Tank size and tube placement allows use of the Dewar at either Cassegrain, Bent-Cassegrain, or Nasmyth telescope mounts without spillage. A nominal fill of $40 \%$ capacity will give at least a two-day hold time.

tendency of the crescent to open when under vacuum, and provide a stable framework for mounting optics. The three inner bulkheads form an integral unit that supports all of the optics components. The two outer bulkheads provide cold volume mounting points for connection to the vacuum vessel.

Fill and vent tube end points within the $\mathrm{LN}_{2}$ tank are placed such that the same design can be utilized at Cassegrain, Bent-Cassegrain and Nasmyth telescope mounts. The $\mathrm{LN}_{2}$ tank is sized such that at least two-day hold times are achieved with cryogens filled to $\sim 40 \%$ capacity. This capacity is defined by the position of the end point of the fill and vent tubes within the tank for which an instrument mounted at any telescope position, with any orientation on the sky and rotator position, will not spill cryogens (Figure 7). An auxiliary $\mathrm{LN}_{2}$ tank will be mounted adjacent to the spectrograph camera to provide temperature control for the spectrograph detector.

The overall Dewar length is 46.5 " and encircled diameter is 32". Empty weight is $\sim 650 \mathrm{lb}$; with controllers boxes and half-load of cryogens the system weight should be $\sim 800 \mathrm{lb}$.

\subsection{Structural Considerations}

Relative movement of the instrument optics due to changing gravity vectors during telescope pointing is minimized by various methods (Figure 8). Radial movement of the cold volume (with respect to the vacuum shell and telescope) is constrained using four pre-stressed hand-wound fiberglass epoxy straps that connect each cylindrical end cap of the vacuum vessel with the outer bulkheads of the cold volume. Axial rotation and fore-and-aft movement of the cold volume (with respect to the vacuum shell and telescope) is also constrained through the use of ' $\mathrm{X}$ ' shaped G-10 connecting tabs on either side of the long axis of the Dewar. This mounting system should hold the $450 \mathrm{lbs}(205 \mathrm{~kg})$ within $1 \mathrm{mil}(25 \mu \mathrm{m})$.

Within the cold volume the crescent shaped tank provides a stable mounting platform for the three inner bulkheads with mounted optical components. The tank and inner bulkhead system should combine to reduce differential movement between the slit and detector to $65 \mu \mathrm{m}$ (1/4 slit width) for all loads during pointing.

\section{STRAY LIGHT}

Stray light from outside the instrument FOV will be controlled by the cold Lyot stop placed at the image of the primary formed by the re-imaging optics prior to the slit. But light that correctly passes through the slit can also generate stray light within the spectrograph channel such as scattering from imperfect optical surfaces. 


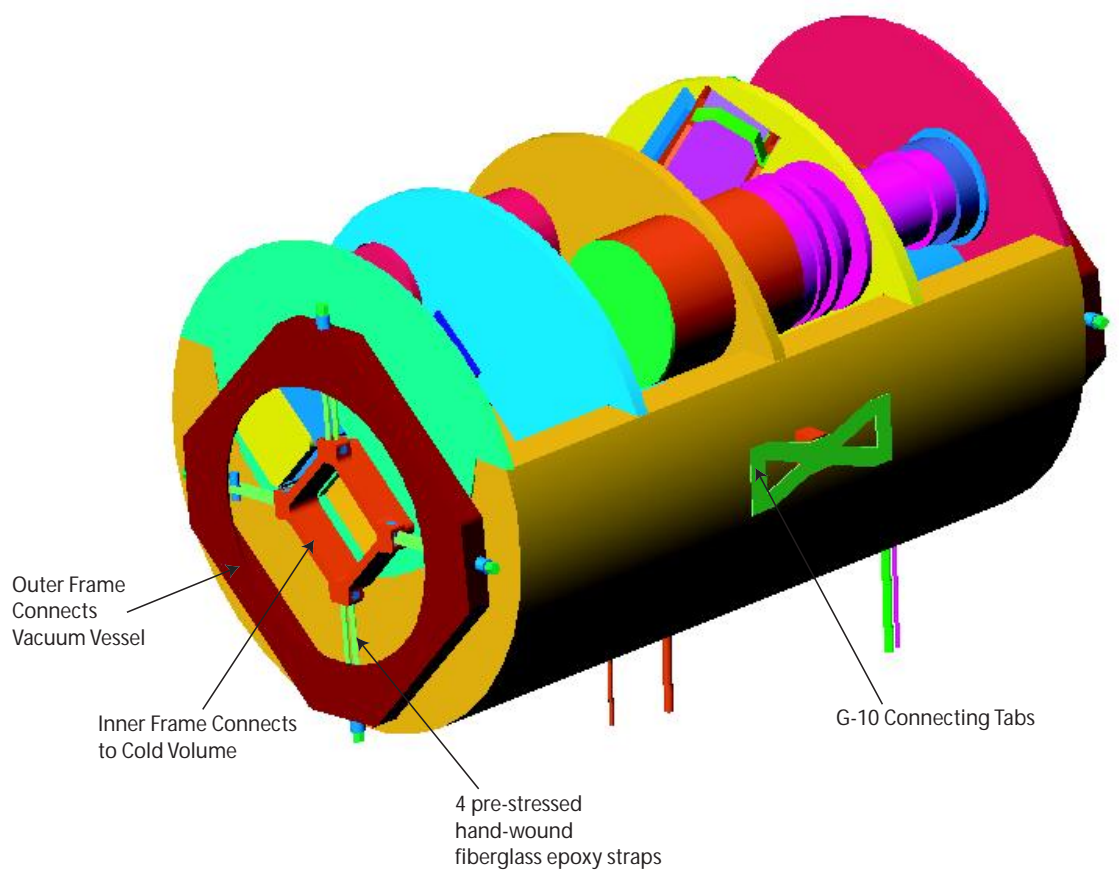

Figure 8. Radial movement of the cold volume is constrained using four pre-stressed hand-wound fiberglass epoxy straps that connect each cylindrical end cap of the vacuum vessel with the outer bulkheads of the cold volume. Axial rotation and fore-and-aft movement of the cold volume is constrained through the use of ' $\mathrm{X}$ ' shaped G-10 connecting tabs on either side of the long axis of the Dewar.

Dispersion of unwanted wavelengths off of the grating is of particular concern. Most insidious from a stray light standpoint are the beams of light diffracted by the grating at wavelengths that come to a focus just off the edge of the detector. At these wavelengths the beams are partially vignetted by the finite sized optics within the spectrograph camera. This vignetting gives rise to scattering from inside edges of baffles and optical element outside edges. In particular, light from the bright thermal infrared between $\lambda=2.46 \mu \mathrm{m}$ (the red edge of order 3 on the detector) and wavelengths shortward of the $\mathrm{HgCdTe}$ cut-off $(\lambda \sim 2.6 \mu \mathrm{m})$ pose the most concern. Not only does light within this wavelength range in order 3 fall just off the edge of the detector. But light at these wavelengths from order 2 also falls just off the opposite corner of the detector. Effective field stops surrounding the detector will be important for preventing this light from entering the detector through the detector edges or through ghost reflections.

We have contracted with Breault Research Organization, Inc. ${ }^{\text {I }}$ to conduct stray light analysis of the spectrograph channel. This study will provide guidance on mechanical stray light mitigation, such as the best position for baffles within the spectrograph camera, effectiveness of grooves within the camera barrel, and the most important places for black paint.

\section{DETECTORS \& ELECTRONICS}

\subsection{Detectors}

Two detectors are used in this instrument. The spectrograph channel will use two science-grade quadrants of a Rockwell Scientific HAWAII-II PACE HgCdTe detector. Thus the active detector size will be 2048 x 1024 pixels. As discussed in Section 5.1, an auxiliary $\mathrm{LN}_{2}$ tank will be used to enable thermal control of the spectrograph

\footnotetext{
๑ Breault Research Organization, Inc., 6400 East Grant Road, Suite \#350 Tucson, Arizona, 85715 USA Tel: 520-7210500 .
} 
detector to a temperature slightly greater than $77 \mathrm{~K}$. This will provide better dark current stability and less zero point drift. The slit viewing channel will fully illuminate a Rockwell Scientific engineering-grade HAWAII-I PACE HgCdTe 1024 x 1024 pixel detector.

\subsection{Electronics \& Software}

The spectrograph and slit viewing detectors will be operated by independent computer systems as well as Generation-III controllers from Astronomical Research Cameras, Inc." with separate grounds and supplies but with a shared common shield (the Dewar). We expect to operate both systems from a common master clock with a software provision to pause the imager to avoid electrical interference with the science readout.

The ArcVIEW software package ${ }^{6}$ will be used for the Palomar instrument. This package will require only minor modifications to support coordinated readout of the two systems, and may be enhanced to provide either guide signals to the telescope control system or a steady stream of sub-images containing the guide star (The slit viewer will be used for guiding at the Palomar 200-inch). ArcVIEW is otherwise already equipped to control these detectors, provide Fowler sampling and/or real time frame coadding, and write FITS format image files.

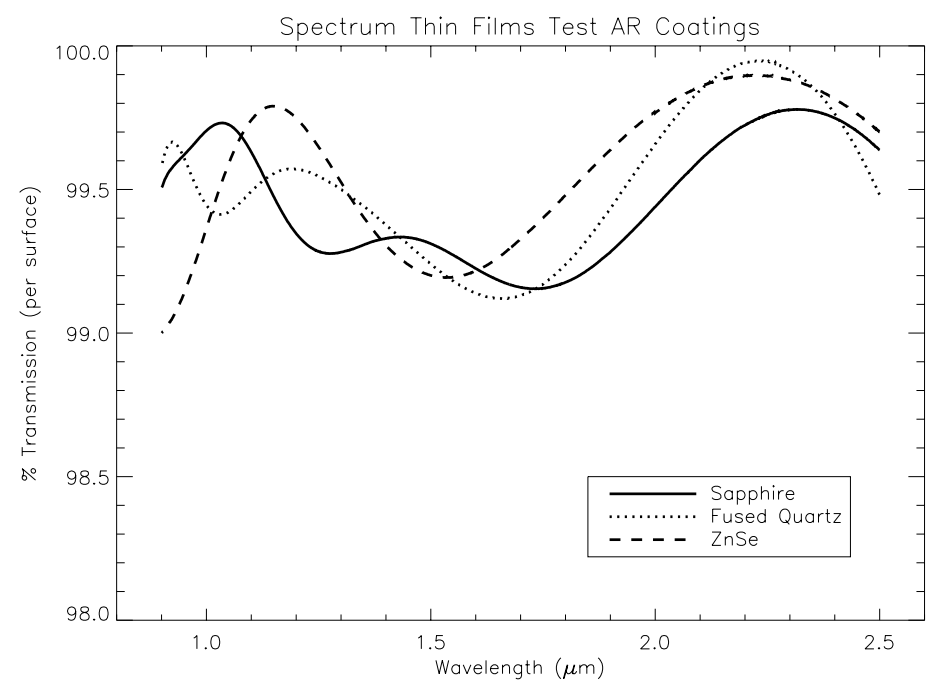

Figure 9. Test AR coatings for Sapphire, Fused Quartz and ZnSe obtained from Spectrum Thin Films. Transmission is for one surface and coatings are designed for normal incidence.

\section{ANTICIPATED SPECTROGRAPH PERFORMANCE}

\subsection{Throughput}

Significant attention has been given to the refractive optics anti-reflection coatings and grating efficiencies to maintain satisfactory throughput. Estimated peak throughput (from telescope primary through detector QE) is $\sim 19 \%, \sim 23 \%$, and $\sim 27 \%$ in $\mathrm{J}, \mathrm{H}$ and $\mathrm{K}$ bands, respectively.

\subsubsection{Anti-Reflection Coatings}

The spectrograph optical train includes the 2-3 telescope mirrors, 22 refractive surfaces, six reflective surfaces, and the detector substrate. To maximize throughput it is imperative that reflection losses be minimized through good performance anti-reflection (AR) coatings. Very promising test AR coatings for Sapphire, Fused Quartz and ZnSe were obtained from Spectrum Thin Films** (Figure 9): their test coatings achieved $>99 \%$ transmission

\footnotetext{
"Astronomical Research Cameras, Inc., 3547 Camino del Rio South, Suite A San Diego, CA 92108 USA, Tel: 619-5847979 .

** Spectrum Thin Films, 100-E Knickerbocker Ave, Bohemia, NY 11716 USA, Tel: 631-589-3502.
} 
between $1.0-2.5 \mu \mathrm{m}$ per surface. Coating performance for $\mathrm{ZnS}$ and $\mathrm{CaF}_{2}$ should be similar to that of ZnSe and Fused Silica, resp. We hope to use coatings with this performance for the Dewar window, spectrograph camera and slit viewing camera elements. Test coatings for the prisms have not been obtained, but we hope to use similar performance coatings for those elements. The detector substrates do not have AR coatings, and cannot be coated after production without risk.

\subsubsection{Grating Efficiency}

The reflection grating has very good efficiency. Peak efficiencies are expected to be $\sim 70 \%, \sim 78 \%$ and $\sim 81 \%$ in J, H, and K bands. These estimates are based on Spectra-Physics efficiency measurements of the grating at near-Littrow use for several orders, and estimated performance losses incurred when the grating is used at $30 \mathrm{deg}$ angular deviation compared to a Littrow configuration using PCGRATE-2 $\mathrm{E}^{\dagger \dagger}$ software.

Table 3. Estimated spectrograph continuum sensitivity for Palomar 200-inch. Assumes $\mathrm{S} / \mathrm{N}=5$ per resolution element, system throughput of $0.17,0.19$ and $0.22 \mathrm{in} \mathrm{J}, \mathrm{H}$ and $\mathrm{K}$, background level between $\mathrm{OH}-$ lines of 70,200 and $70 \mu \mathrm{Jy} / \mathrm{arcsec}^{2}$ in $\mathrm{J}, \mathrm{H}$, and $\mathrm{K}$ bands, $\mathrm{RN}=5 \mathrm{e}^{-}$and $\mathrm{i}_{\mathrm{dark}}=0.01 \mathrm{e}^{-} / \mathrm{sec}$.

\begin{tabular}{|c|c|c|c|}
\hline Band & $600 \mathrm{sec}$ & $1800 \mathrm{sec}$ & $3600 \mathrm{sec}$ \\
\hline $\mathrm{J}$ & 19.6 & 20.3 & 20.7 \\
\hline $\mathrm{H}$ & 18.8 & 19.4 & 19.8 \\
\hline $\mathrm{K}$ & 17.7 & 18.3 & 18.7 \\
\hline
\end{tabular}

\subsection{Sensitivity}

Sensitivity estimates for the spectrograph at the Palomar 200-inch are listed in Table 3. The estimates assume $\mathrm{S} / \mathrm{N}=5$ per resolution element, system throughput of $0.17,0.19$ and $0.22 \mathrm{in} \mathrm{J}, \mathrm{H}$ and $\mathrm{K}$, background level between $\mathrm{OH}$-lines of 70, 200 and $70 \mu \mathrm{Jy} / \operatorname{arcsec}^{2}$ in $\mathrm{J}, \mathrm{H}$, and $\mathrm{K}$ bands, $\mathrm{RN}=5 \mathrm{e}^{-}$and $\mathrm{i}_{\text {dark }}=0.01 \mathrm{e}^{-} / \mathrm{sec}$.

\section{ACKNOWLEDGMENTS}

Funding for the University of Virginia instrument comes in large part from a generous gift from the F.H. Levinson Fund of the Peninsula Community Foundation.

\section{REFERENCES}

1. M. F. Skrutskie et al., "The Two Micron All Sky Survey (2MASS): Overview and Status," in The Impact of Large Scale Near-IR Sky Surveys, F. Garzon et al., eds., p. 25, Kluwer Academic, Dordrecht, 1997.

2. D. G. York et al., "The Sloan Digital Sky Survey: Technical Summary," AJ 120, p. 1579, 2000.

3. M. W. Werner et al., "The Spitzer Space Telescope Mission," ApJS in press, 2004.

4. P. Martini and D. L. DePoy, "Optimal resolutions for IR spectroscopy through the OH Airglow," in Optical and IR Telescope Instrumentation and Detectors, M. Iye and A. F. Moorwood, eds., Proc. SPIE 4008, pp. 695-702, 2000.

5. J. H. Elias et al., "Design of the Gemini near-infrared spectrometer," in Infrared Astronomical Instrumentation, A. M. Fowler, ed., Proc. SPIE 3354, pp. 555-565, 1998.

6. M. C. Ashe, M. Bonati, and S. Heathcote, "ArcVIEW: a LabVIEW-based Astronomical Instrumentation System," in Telescope and Instrumentation Control Software II, H. Lewis, ed., Proc. SPIE 4848, pp. 508$518,2002$.

\footnotetext{
${ }^{\dagger \dagger}$ International Intellectual Group, Inc., Rochester, NY, USA, Tel: 585-218-9829.
} 